\title{
MULTIWAVELENGTH VARIABILITY IN THE SYMBIOTIC NOVA V1016 CYG
}

\author{
Ś. Parimucha ${ }^{1}$ and M. Vaňko ${ }^{2}$
}

Analysis of the optical and infrared photometry together with UV spectroscopy led to discovery of the 15-years periodicity in the symbiotic system V1016 Cyg. This period could be interpreted as a orbital period in the binary system consisting of the Mira variable and the hot white dwarf.

We have analyzed long-term optical photographic and $U B V$ photoelectric photometry of V1016 Cyg. Collected observations cover pre- and post-outburst stages of the system. The light curve suggests four stages of activity: the pre-outburst flare in 1949 , the main nova-like outburst in 1964, and two postoutbursts, decreasing-amplitude flares in 1980 and 1994. merectively. Activity episodes affecting the system repeat with an interval of $\sim 15$ years. The ephemeris for the ac tivity maxima is (see Parimucha et al., 2000)

$$
J D_{m a, r}=(2127590 \pm 250)+(5510 \pm 90) \times E .
$$

We have also collected all available infrared $J H K$ photometric observations. Their list could be found in Parimucha (2003). Period analysis improved the period of pulsations of the Mira component to $474 \pm 2$ day: According to Whitclock (1987), the $J-K$ color index in symbiotic Miras is little affected by the Mira type variable pulsation, but is very sensitive to the amount of circumstellar dust around the cool component. Our stuly of the $J-K$ color index of the V1016 ('yg shows that it had a maximum in 1988 and a clear minimum in 1992, together with a more uncertain maximum (in 1973) and a further possible minimum (in 1977). This suggest 15-years periodicity in color index variations, which is similar to the period detected from optical photometry. A short, but strong dust formation episode in 1983 with amplitude $\sim 1 \mathrm{mag}$ (reported by Taranova \& Yudin. 1986), which occurred three years after the observed maxima both in the U passband and space-borne UV (see below). can be interpreted as a dust formation in the ejecta of the srmbiotic nova.

\footnotetext{
${ }^{1}$ Institute of Physics, Faculty of Natural Sciences, Iniversity of P. J. Safárik, Moyzesova 16, 04001 Košice, The Slovak Republic (parimuchoita3.sk).

${ }^{2}$ Astr. Institute of the Slovak Academy of Sciences, 05960 Tatranská Lomnica, The Slovak Republic (vanko@ta3.sk).
}

The ultraviolet spectroscopy observations of V1016 Cyg was performed by IUE satellite from 1978 to 1995 . One UV spectrum was taken by HUT in 1995. We have used calibrated low and high dispersion IUE and HUT spectra provided by INES and MAST archives. Spectra were corrected to interstellar extinction by $\mathrm{E}(\mathrm{B}-\mathrm{V})=0.28$ (Nussbaumer \& Schild, 1981) and were very critically measured. We have determined continuum fluxes at $\lambda 1840 \AA$ and $\lambda 2530 \AA$ and fluxes of the collisionally excited emission lines OIII] $\lambda 1661 \AA$, OIII] $\lambda 1666 \AA$, NIII $] \lambda 1750 \AA$, SiIII] $\lambda 1892 \AA$ and CIII] $\lambda 1909 \AA$. The evolution of both UV continua and emission lines fluxes from 1978 to 1996 is characterized by two maxima matching the activity epochs shown by optical photometry. The second maximum is not sufficiently covered, but it seems to be less intensive than the first one. A detailed inspection of these variations shows 410-days delay of the maximum of UV emission lines fluxes with respect to the maximum of continuum, which agree well with the maximum determined from ephemeris (1). It can be seen that major dust obscuration in 1983 reflects in a recognizable flux drop of the majority of UV emission lines in 1983-1984.

Analysis of the multiwavelength variability in the symbiotic system V1016 Cyg led to the discovery of the 15-years periodicity. This can be interpreted as a orbital period in a binary with moderate elliptical orbit. Observed flares can be explained by enhanced mass transfer from the Mira component during periastron passage. The main outburst occurred in 1964 when critical amount of material had been gradually accumulated. This eruption led to the creation of the complex nebula visible mainly in radio wavelengths.

This work was supported by VEGA Grant $2 / 1157$ of the Slovak Academy of Sciences and grant VVGS/014/2003/F of the University of P.J. Safárik, Košice.

\section{REFERENCES}

Nussbaumer, H., \& Schild, H. 1981. A\&A, 101. 118

Parimucha, S.. Arkhipova, V. P., Chochol, D., et al., 2000, CAOSP, 30, 99

Parimucha, S. 2003, CAOSP, 33, 99

Taranova, O. G., \& Yudin, B. F. 1986, AZh, 63, 317

Whitelock, P. A. 1987, PASP, 99, 573 